\title{
UNCERTAINTY EVALUATION IN CRITICALITY CALCULATIONS USING THE TSUNAMI METHODOLOGY
}

\author{
MATĚJ ŠIKL ${ }^{a, b, *}$, FRANTIŠEK HAVLŮ ${ }^{b}$ \\ ${ }^{a}$ Department of Nuclear Reactors, Faculty of Nuclear Sciences and Physical Engineering, Czech Technical \\ University in Prague, V Holesovickach 2, Prague 8, CZ \\ ${ }^{b}$ Department of Reactor Physics and Fuel Cycle Support, Nuclear Research Institute Rez near Prague, CZ \\ * corresponding author: siklmate@fjfi.cvut.cz
}

\begin{abstract}
Procedure of criticality calculations and uncertainty evaluations currently has several insufficiencies which could lead to potential non-conservativeness. Paper discuss and describes possibilities of elimination of these insufficiencies and thereby ensuring safely sub-critical results of calculations. Selection of experiments for validation based on similarity coefficients between systems acquired from TSUNAMI-IP is used for this purpose. TSUNAMI-IP operates with sensitivity of multiplication factor to cross sections of individual isotopes and reactions between compared systems. Moreover, TSUNAMI-IP determines uncertainty of calculations brought in using insufficiently similar experiments. Within research, effects of variable parameters used in calculations inputs are investigated. In conclusion optimized suggested approach for validations and uncertainty evaluation using TSUNAMI module from SCALE based on knowledge acquired within research is described.
\end{abstract}

KEYWORDS: validation, subcriticality, TSUNAMI, similarity between systems, penalty, pool storage.

\section{INTRODUCTION}

Sources of uncertainties are contained in current approach for criticality calculations. Most of these uncertainties are considered in uncertainty evaluation, even so some of them remain and are not evaluated. It could possibly lead to non-conservative evaluation and even to violation of safety limits.

Computational code for criticality calculations is validated based on specific types of critical experiments. Thereafter is said that code gives correct believable results. This paper focuses to selection of experiments used for validation, because main omitted source of uncertainties is contained there.

One purpose for validating codes at Department of Reactor Physics (NRI Rez) are calculations of spent fuel storages. Code is usually validated using critical experiments with fresh fuel. However when code is applicated to spent fuel storage there is not considered effect of disparity between used experiments and newly suggested spent fuel storage. This brings potential risk and non-conservativeness described above.

Goal to be achieved was to evaluate effect of disparity and prepare instructions for validating code application on spent fuel systems.

Whole research was done by means of SCALE [1] with advantage of it's module TSUNAMI (uses KENO Monte Carlo code, will be described later).

\section{Current process of validation AND UNCERTAINTY EVALUATION}

Process of validation and uncertainty evaluation in criticality calculations is currently carried out this way:
(1.) firstly set of critical experiments for validation is chosen - selection usually depends on which experiments are available

- for each experiment $(i)$ are known both $k_{\text {eff }}-$ measured $\left(k_{\mathrm{m}}^{\mathrm{e}, i}\right)$ and calculated $\left(k_{\mathrm{c}}^{\mathrm{e}, i}\right)$

(2.) then biases $b_{i}$ for each experiment $(i)$ are calculated

(a) $b_{i}=k_{\mathrm{m}}^{\mathrm{e}, i}-k_{\mathrm{c}}^{\mathrm{e}, i}$, if $k_{\mathrm{c}}^{\mathrm{e}, i}<k_{\mathrm{m}}^{\mathrm{e}, i}$

(b) $b_{i}=0$, if $k_{\mathrm{c}}^{\mathrm{e}, i}>=k_{\mathrm{m}}^{\mathrm{e}, i}$

(3.) average bias is calculated from $b_{i}$

(4.) model of new system, which is newly designed (called application), is calculated $\rightarrow k_{\mathrm{c}}^{\mathrm{a}} \pm \sigma_{k_{\mathrm{c}}^{\mathrm{a}}}$

- where $\sigma_{k_{\mathrm{c}}}$ is statistic error of KENO-VI Monte Carlo calculation

(5.) finally sum of

$$
k_{\mathrm{c}}^{\mathrm{a}}+2 \sigma_{k_{\mathrm{c}}^{\mathrm{a}}}+\text { bias }+2 \sigma_{\text {bias }}
$$

must be smaller than required safety limit (usually set by legislation)

As was already pointed out above, in this procedure disparity between experiments and applications is not considered. It affect mainly two parts of the procedure:

(1.) experiments for evaluation of bias are chosen without consideration of similarity between the application and the experiment

(2.) in the final equation there is no term expressing and quantifying potential deviation caused by disparity between experiment and application 
Both of these aspects can be resolved using TSUNAMI.

\section{TSUNAMI}

TSUNAMI is part of complex computational code SCALE and its name stands for Tools for Sensitivity and Uncertainty Analysis Methodology Implementation. Complete documentation can be found in 1 .

When calculating systems parameters using TSUNAMI-3D-K6 (Monte Carlo code KENO is used for these calculations), one of the possible outputs is the sensitivity data file (.sdf). These contains sensitivity informations (how much $k_{\text {eff }}$ changes because of change of cross sections $\left.-\frac{\delta \mathrm{k}}{\delta \sigma}\right)$ for each nuclide, energy group and reaction. From these files TSUNAMI-IP (Indices and Parameters) calculates amount of similarity between chosen systems. Several similarity coefficient exist, which are calculated different ways based on sensitivities and uncertainties of cross section determination.

Most important coefficient, based on both sensitivity and uncertainty is $c_{\mathrm{k}}$. It expresses overall similarity of two systems (detailed description can be found in 11). Coefficient $c_{\mathrm{k}}$ is normalized such way, that similar systems have $c_{\mathrm{k}}=1$ and totally dissimilar systems have $c_{\mathrm{k}}=0$. Currently similarity with $c_{\mathrm{k}}>0.95$ is considered sufficient.

Second important value is penalty. It is calculated based on both sensitivity and uncertainty. Penalty expresses in $\% \quad \frac{\Delta \mathrm{k}}{\mathrm{k}}$ uncertainty of calculating application system based on dissimilarity between experiments and applications. When calculating penalty it is possible to choose which experiments should or should not be used for penalty assessment. Parameter cvalue can be set to ensure that only experiments with $c_{\mathrm{k}}>$ cvalue will be used for penalty evaluation. Using cvalue is necessary - it is not required to deduct informations from totally dissimilar systems.

Each system has maximum value for penalty, which responds with relative standard deviation of $k_{\text {eff }}$ due to cross-section covariance data. Usually in results of various systems calculations observed maximal penalty was around $0.6 \% \frac{\Delta \mathrm{k}}{\mathrm{k}}$, which corresponds with 2. Value of maximum penalty is included as "relative standard deviation of $k_{\text {eff }}$ due to cross-section covariance data" in output, when system is calculated with TSUNAMI-3D-K6 module from SCALE.

Diagram of entire process of calculations and analysis using TSUNAMI is shown in figure 1 .

\section{Comparing SYSTEM SIMILARITY AND VALIDATION QUALITY FOR} VARIOUS TYPES OF EXPERIMENTS

For further usage determination of behaviour of similarity coefficient was needed. Firstly several pincell systems with varying parameters were

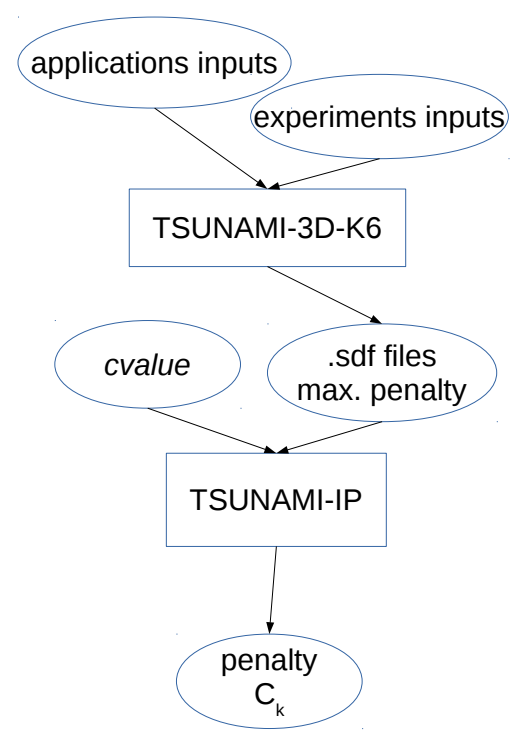

Figure 1. Diagram of evaluation process using TSUNAMI

compared for this purpose. Later, spent fuel wet storages as applications and different types of experiments were examined.

\subsection{SimPLIFIED SYSTEMS}

Infinite systems of fuel rods were modelled. For comparison some parameters within systems were changed:

(1.) grid type

(2.) enrichment

(3.) pitch

(4.) amount of absorber $\left(\mathrm{H}_{3} \mathrm{BO}_{3}\right)$

(5.) isotopic composition of fuel due to burnup.

In each case only one parameter was changed, others remained nominal, which means:

- enrichment $4 \%$

- pitch between fuel rods $0.61 \mathrm{~cm}$

- $4 \mathrm{~g} / \mathrm{kg}$ of $\mathrm{H}_{3} \mathrm{BO}_{3}$ in moderator.

When comparing triangular and square grid the moderator-to-fuel ratio for compared systems remained unchanged. Multiples from 0.8 to 2.5 of nominal pitch between fuel rods were used in infinite grids. For each multiple of nominal pitch $c_{\mathrm{k}}$ between triangular and square grid was bigger than 0.99 , thus the experiments with square grid can be used for validation for calculating triangular grids and same the other way.

After comparison of square and triangular pitch and proving their similarity, all further comparisons were done for triangular grid.

Comparison of similarity between systems with different enrichments is displayed in figure 2. System with enrichment $4 \%$ was used as the experiment and 


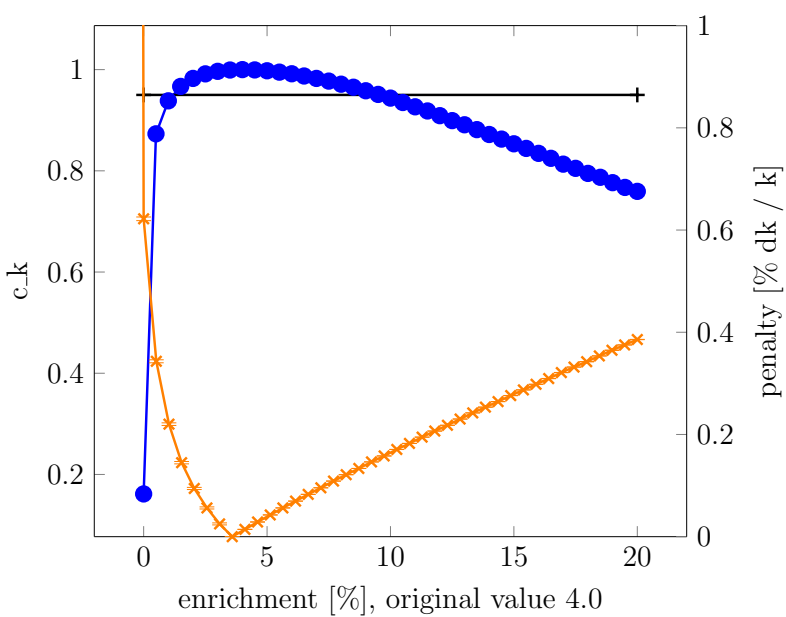

$-\mathrm{c} \_\mathrm{k}+\mathrm{c} \_\mathrm{k}=0.95 *$ penalty

FIGURE 2. Evaluation of similarity between systems with different enrichments.

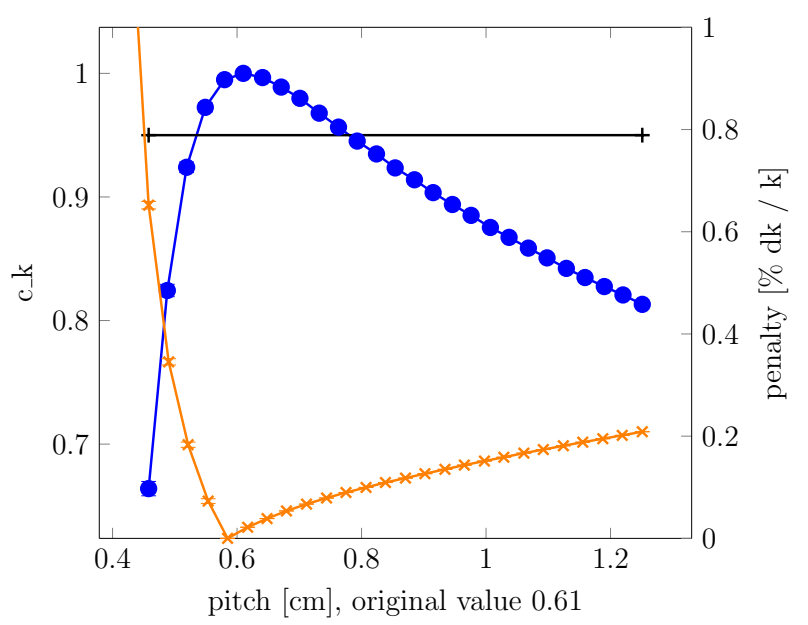

$-\mathrm{c} \_\mathrm{k} \longrightarrow \mathrm{c} \_\mathrm{k}=0.95 \rightarrow$ penalty

FIGURE 3. Evaluation of similarity between systems with different pitches.

applications had from 0 to $20 \%$ enrichment. In figure 2 it can be seen that variation in enrichment from 1.5 to $8 \%$ has still $c_{\mathrm{k}}>0.95$. That seems to be a sufficient range for a typical application.

Figure 3 shows comparison between systems with different pitches. Grid with nominal pitch $0.61 \mathrm{~cm}$ was taken as the experiment. As can be seen in figure 3 systems with changes in pitch smaller than $0.1 \mathrm{~cm}$ are still sufficiently similar. Value of $0.1 \mathrm{~cm}$ seems to be small variability, but in fact is enough, because experiments will be typically available for identical or similar types of fuel assemblies as in the applications.

Boron acid amount in moderator was next parameter changed. System with $4 \mathrm{~g} / \mathrm{kg}$ boron acid in moderator was taken as the experiment. Figure 4 shows that comparison and it can be seen that $c_{\mathrm{k}}$ remains bigger than 0.99 for all applications. This fact is important, because it means it is possible to use

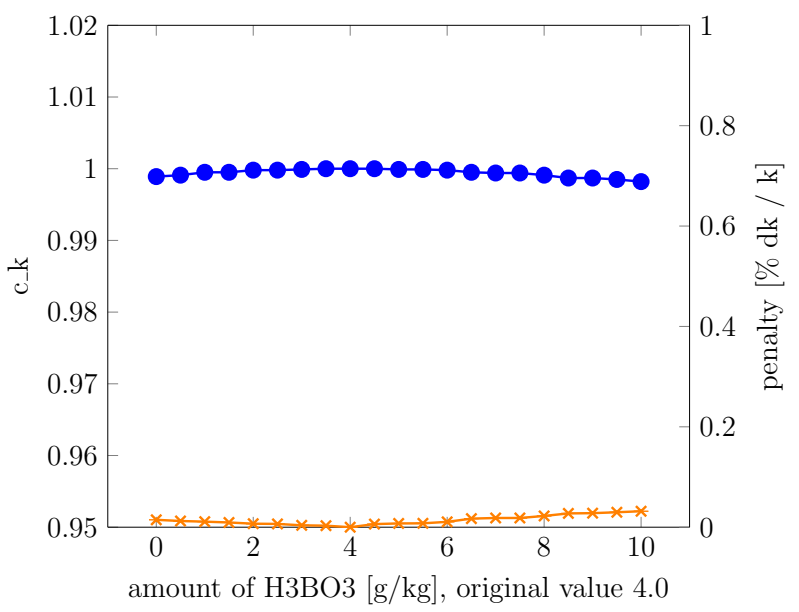

$\mathrm{c}_{-} \mathrm{k} *$ penalty

FigURE 4. Evaluation of similarity between systems with different amount of $\mathrm{H}_{3} \mathrm{BO}_{3}$ in moderator.

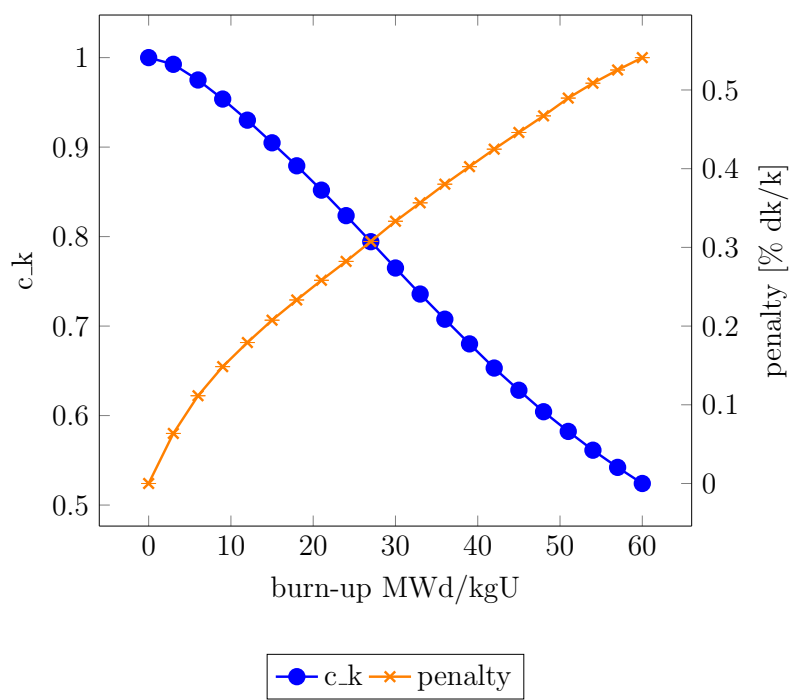

Figure 5. Comparison of irradiated fuel with fresh $\mathrm{UO}_{2}$ fuel.

experiment without boron for application including boron with no big impact.

Later, systems with fuel rods in different degrees of irradiation were calculated and compared. To get closer to reality systems with fresh fuel only were used as experiments, because no experiments with irradiated fuel are included in evaluated databases of critical experiments.

Applications are systems with isotopic composition obtained with ORIGEN, input for ORIGEN was fresh $\mathrm{UO}_{2}$ fuel.

Firstly irradiated fuel applications were compared with fresh $\mathrm{UO}_{2}$ fuel as the experiment and is shown in figure 5 . In figure 5 can be seen that if $c_{\mathrm{k}}$ will be required bigger than 0.95 , only spent fuel with irradiation smaller than approximately $10 \mathrm{MWd} / \mathrm{kgU}$ are sufficiently similar. 


\begin{tabular}{cc}
\hline element & atom density \\
\hline $\mathrm{pu}-238$ & $7.39 \mathrm{E}-06$ \\
$\mathrm{pu}-239$ & $4.10 \mathrm{E}-03$ \\
$\mathrm{pu}-240$ & $5.61 \mathrm{E}-04$ \\
$\mathrm{pu}-241$ & $4.38 \mathrm{E}-05$ \\
$\mathrm{pu}-242$ & $1.70 \mathrm{E}-05$ \\
$\mathrm{u}-235$ & $1.20 \mathrm{E}-04$ \\
$\mathrm{u}-238$ & $1.69 \mathrm{E}-02$ \\
$\mathrm{o}$ & $4.37 \mathrm{E}-02$ \\
$\mathrm{am}-241$ & $3.43 \mathrm{E}-05$ \\
\hline
\end{tabular}

TABLE 1. Fresh MOX material composition.

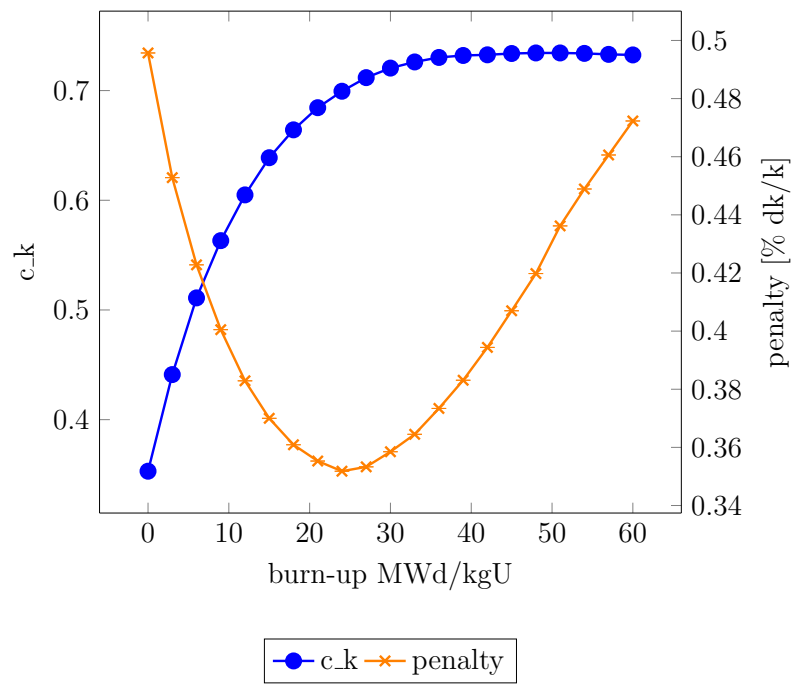

FiguRE 6. Comparison of irradiated fuel with fresh MOX fuel.

Secondly fresh MOX fuel was used as the experiment. MOX material composition used in calculations follows in table 1 .

MOX was chosen because during burnup actinides are created (most important is $\mathrm{Pu}$ ) and in MOX fuel $\mathrm{Pu}$ and $\mathrm{Am}$ isotopes are contained. Output of comparison is shown in figure 6 and interesting progress in $c_{\mathrm{k}}$ can be observed. Applications with small burnup are not similar with experiment $\left(c_{\mathrm{k}}<\right.$ $0.5)$, but later with burnup raises to $c_{\mathrm{k}}=0.7$.

This progress led to comparing applications (irradiated fuel) with both experiments $\left(\mathrm{UO}_{2}\right.$ and MOX) in one time. This comparison is shown in figure 7

Figure 7 brings one essential fact: $c_{\mathrm{k}}>0.95$ is currently unfulfillable requirement.

\subsection{ReAlistic SYSTEMS}

Later more realistic systems were calculated, after first simplified comparison was completed.

Experiments were taken from International Handbook of Evaluated Criticality Safety Benchmark Experiments (ICSBEP, [3]) database. This database collects nearly 5000 critical experiment and benchmarks from nuclear facilities around the world.

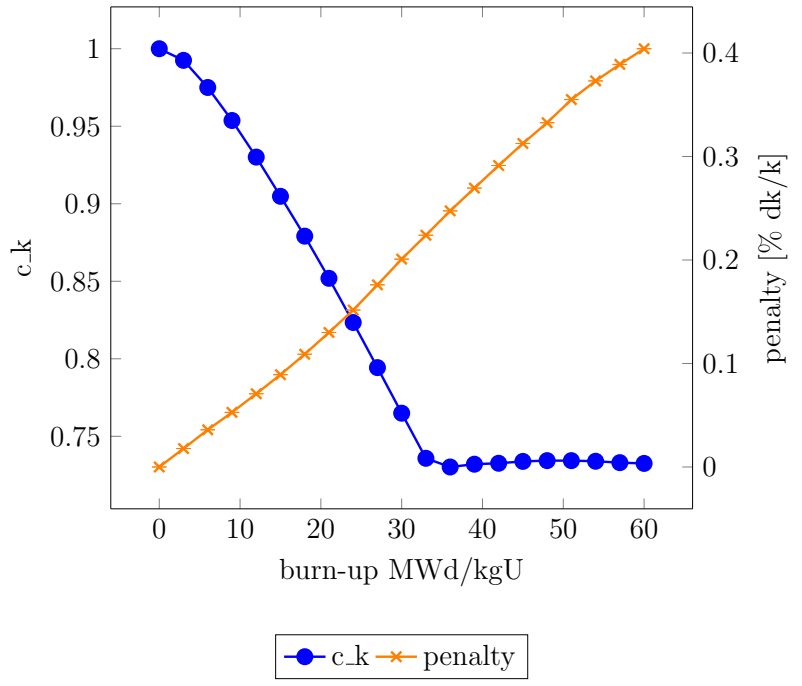

Figure 7. Comparison of irradiated fuel with fresh $\mathrm{UO}_{2}$ and MOX fuel experiments together.

Systems with low-, intermediate- and highly- enriched uranium, MOX and other types of fuel are included. For some experiments are available input files (SCALE or MCNP) and for some are available .sdf files. For following comparison .sdf files from there were used, even though they were produced with another version of nuclear data libraries. This was done to save computational time and for possibility for using hundreds of experiments.

Applications were systems representing an infinite lattice of Dukovany nuclear power plant wet spent fuel storage cells with varying fuel burnup. In this wet spent fuel storage two racks are located - the upper and the lower, where the lower one has boron metal sheets placed between fuel assembly positions. For the application a fuel assembly Gd2M with mean enrichment $4.38 \%$ with burnup from 0 to $60 \mathrm{MWd} / \mathrm{kgU}$ was chosen.

Maximum possible penalty for the applications in question ranges from 0.4 to $0.6 \% \frac{\Delta \mathrm{k}}{\mathrm{k}}$.

As it was said, variable types of experiments were available and comparison was performed with various combinations of experiments. Results of comparison were poor for application with small burnup when experiments were MOX and for applications with high burnup when experiments were fresh low enriched uranium fuel, which corresponds with results from section 4.1. Therefore result for comparison with mix of available experiments with low enriched uranium and MOX will be discussed.

First, comparison with variable cvalue (different for each application) was performed. In the first run $c_{\mathrm{k}}$ were calculated and from these the cvalue for each application was set as 0.75 multiple of the highest $c_{\mathrm{k}}$ achieved for individual application. Value 0.75 was chosen because of two reasons. Firstly experiment filtration is necessary, so only adequate system remains and are used in analysis. Secondly acceptable amount of experiments is needed, so not to filter majority 


\begin{tabular}{cccc}
\hline grid, $\frac{\mathrm{MWd}}{\mathrm{kgU}}$ & cvalue & penalty $\left[\% \frac{\Delta \mathrm{k}}{\mathrm{k}}\right]$ & cvalue passed \\
\hline bottom, 0 & 0.68 & $3.24 \mathrm{E}-2$ & $402 / 726$ \\
bottom, 15 & 0.62 & $3.88 \mathrm{E}-2$ & $489 / 726$ \\
bottom, 30 & 0.58 & $6.48 \mathrm{E}-2$ & $489 / 726$ \\
bottom, 45 & 0.59 & $1.52 \mathrm{E}-1$ & $219 / 726$ \\
bottom, 60 & 0.57 & $2.09 \mathrm{E}-1$ & $228 / 726$ \\
upper, 0 & 0.70 & $2.24 \mathrm{E}-2$ & $359 / 726$ \\
upper, 15 & 0.64 & $5.13 \mathrm{E}-2$ & $395 / 726$ \\
upper, 30 & 0.59 & $1.05 \mathrm{E}-1$ & $291 / 726$ \\
upper, 45 & 0.58 & $2.02 \mathrm{E}-1$ & $164 / 726$ \\
upper, 60 & 0.55 & $2.79 \mathrm{E}-1$ & $166 / 726$ \\
\hline
\end{tabular}

passed through cvlaue test is on one side small, on the other side not all of the experiments were filtered.

This process of calculating and validating seems to be best of all tried. For spent fuel applications MOX and low enriched uranium experiments will be needed.

In next section optimized recommended approach for validating will be described.

\section{RECOMMENDED OPTIMIZED PROCESS}

Based on research realized and knowledge from papers [4] and [5] an optimized validation and uncertainty evaluation in criticality calculations process will be

TABlE 2. Comparison of wet spent fuel storage applications with low enriched uranium and MOX fuel experiments, variable cvalue.

\begin{tabular}{cccc}
\hline grid, $\frac{\mathrm{MWd}}{\mathrm{kgU}}$ & $c_{\mathrm{k}}^{\max }$ & penalty $\left[\% \frac{\Delta \mathrm{k}}{\mathrm{k}}\right]$ & cvalue passed \\
\hline bottom, 0 & 0.90 & $3.28 \mathrm{E}-2$ & $401 / 726$ \\
bottom, 15 & 0.82 & $1.70 \mathrm{E}-1$ & $367 / 726$ \\
bottom, 30 & 0.77 & $1.56 \mathrm{E}-1$ & $139 / 726$ \\
bottom, 45 & 0.79 & $1.57 \mathrm{E}-1$ & $139 / 726$ \\
bottom, 60 & 0.76 & $2.17 \mathrm{E}-1$ & $82 / 726$ \\
upper, 0 & 0.93 & $2.24 \mathrm{E}-2$ & $358 / 726$ \\
upper, 15 & 0.85 & $5.37 \mathrm{E}-2$ & $273 / 726$ \\
upper, 30 & 0.79 & $1.11 \mathrm{E}-1$ & $67 / 726$ \\
upper, 45 & 0.78 & $2.18 \mathrm{E}-1$ & $64 / 726$ \\
upper, 60 & 0.73 & $3.09 \mathrm{E}-1$ & $7 / 726$ \\
\hline
\end{tabular}

TABlE 3. Comparison of wet spent fuel storage applications with low enriched uranium and MOX fuel experiments, cvalue $=0.7$ fixed.

of all experiments is required. Multiplication by 0.75 seemed to fulfil both requirements. The results of comparison of both grids and various levels of irradiation as applications and both together low enriched uranium and MOX fuels as experiments are recorded in table 2

In comparison in table 2 for each application penalty value is smaller than maximum penalty. Standard deviations of the penalties were approximately 2 orders smaller than the penalty values. As expected (because of choice of multiplicative factor) for each application some experiments passed cvalue test. On the other side cvalue for some applications was approaching 0.5 , which is unacceptably low value, hence another system for setting cvalue had to be used. That system has to be independent on application's maximal $c_{\mathrm{k}}$.

Paper [4] deals with requirements cvalue should fulfil. In accordance with that paper cvalue $=0.7$ was fixed in further investigations. Results can be seen in table 3 In table 3 standard deviations of the penalties were approximately 2 orders smaller than the penalty values for each application.

Last comparison (results in table 3) still shows good results for penalty, its value is multiply smaller than value of maximum penalty. Number of experiments (a) 0.7 for cvalue test is based on 4, requirement for at least 40 critical experiments passing cvalue test is based on 5 .

As it was said in introduction, main issues in the current approach for validation are the way for choosing experiments for determining bias and the neglection if dissimilarity between experiments used in validation and applications. Both of these can be resolved using TSUNAMI.

Firstly, TSUNAMI-IP analysis should be done for experiments considered for use in bias evaluation. The choice of experiments should be:

- if at least 40 experiments with $c_{\mathrm{k}}>0.7$ to application will be found $\rightarrow$ these experiments should be used for bias determination

- if less than 40 experiments with such similarity will be found $\rightarrow 40$ experiments most similar (with highest $c_{\mathrm{k}}$ ) should be used for determinating bias.

For determining the value of penalty the following approach should be used:

- if at least 40 experiments with $c_{\mathrm{k}}>0.7$ to application will be found $\rightarrow$ value of penalty obtained from TSUNAMI can be used

- if less than 40 experiments with such similarity will be found $\rightarrow$ value of maximum penalty should be used.

With this approach, the equation 1 is changed to

$k_{\mathrm{c}}^{\mathrm{a}}+2 \sigma_{k_{\mathrm{c}}^{\mathrm{a}}}+$ bias $+2 \sigma_{\text {bias }}+$ penalty $+2 \sigma_{\text {penalty }}<U S L$,

where $U S L$ stands for upper safety limit.

\section{Conclusion}

The issues in the current approach for validating and uncertainty evaluation in criticality calculation were pointed out and goal to resolve these was set. When evaluating criticality calculations, many sources of uncertainties are included, but influence of dissimilarity between system is currently neglected.

This influence could be evaluated using TSUNAMI methodology. For these purpose TSUNAMI was described and calculations were performed. From these calculations possible ranges of individual parameters for sufficient similarity were determined. 
Moreover recommendation for selection of critical experiment used for validation was found.

All of these experiences were used to produce a recommended approach described in section 5 where easy to follow guide is placed. During the whole research and preparing recommended approach an effort to optimization was carried out, hence recommended approach has not too strict requirements while requesting all important inputs and being conservatively safe.

Correctly performed procedure can minimize deviation due to disparity of systems to level of calculation statistic error.

The goal of preparing a complex approach for validating and uncertainty evaluation in criticality calculation was fulfilled and it was done in such way that it is possible to prepare automatic script for processing.

\section{REFERENCES}

[1] B. T. Rearden, M. A. Jessee. SCALE Code System, ORNL/TM-2005/39, Version 6.2.2. Oak Ridge National Laboratory, Oak Ridge, Tennessee, 2017.

[2] M. Klein, L. Gallner, B. Krzykacz-Hausmann, et al. Influence of nuclear data uncertainties on reactor core calculations. Kerntechnik 76:174-178, 2011.

[3] International Handbook of Evaluated Criticality Safety Benchmark Experiments, 2016. NEA/NSC/DOC(95)03, OECD, NEA No. 7328 .

[4] B. Rearden, M. Williams, M. Anderson Jessee, et al. Sensitivity and uncertainty analysis capabilities and data in scale. Nuclear Technology 174:236-288, 2011.

[5] B. L. Broadhead, B. Rearden, C. Hopper, et al. Sensitivity- and uncertainty-based criticality safety validation techniques. Nuclear Science and Engineering 146:340-366, 2004. 